Journal de la Société des américanistes

Journal de la Société

des américanistes

tome $90, \mathrm{n}^{\circ} 1$

\title{
Nouvelles considérations sur les Algonquins et le totémisme
}

Emmanuel Désveaux

\section{(2) OpenEdition}

Journals

Édition électronique

URL : https://journals.openedition.org/jsa/360

DOI : $10.4000 /$ jsa. 360

ISSN : 1957-7842

Éditeur

Société des américanistes

Édition imprimée

Date de publication : 5 janvier 2004

Pagination : 7-24

ISSN : 0037-9174

\section{Référence électronique}

Emmanuel Désveaux, "Nouvelles considérations sur les Algonquins et le totémisme », Journal de la Société des américanistes [En ligne], 90-1 | 2004, mis en ligne le 05 janvier 2009, consulté le 04 septembre 2022. URL : http://journals.openedition.org/jsa/360; DOI : https://doi.org/10.4000/jsa.360 


\title{
NOUVELLES CONSIDÉRATIONS SUR LES ALGONQUINS ET LE TOTÉMISME
}

\author{
Emmanuel DÉSVEAUX * \\ "Whether, this is to say, we are comparing cultural realities, \\ or merely figments of our logical mode of classification. "
}

Robert H. Lowie (1912)

La parution récente d'un article étrangement critique à l'égard du schéma avancé pour la première fois dans Le Totémisme aujourd'hui est l'occasion de revenir sur la table des permutations qu'avait proposée en son temps Lévi-Strauss. Le présent article montre que les quatre combinaisons possibles sont présentes dans le domaine algonquin. Dès lors, l'idée d'un système " totémique » complet algonquin fait sens. Ayant recours au groupe de Klein et à la formule canonique des mythes, il l'établit et en conclut que le déni de la substance en constitue le fondement sémantique. S'ensuivent diverses considérations, à l'échelle régionale puis continentale. [Mots CLÉs : totémisme, LéviStrauss, groupe de Klein, formule canonique des mythes, Ojibwa, substance.]

New considerations about Algonquian people and totemism. The recent publication of a paper which oddly attacks the general pattern imagined for the first time by LéviStrauss in The Totemism today provides an opportunity for a new reappraisal of the famous table of permutations which lays at the core of this book. This paper shows that the four combinations occur in the Algonquin area. This observation leads to the idea of building a full Algonquin « totemic » system, that is finaly achieved using a Klein's group and the canonical formula of myths. The semantical outcome of these operations is a deny of substance. Various considerations follow, adressed at the regional and the continental scales. [KEY wORDS : totemism, Klein's group, canonical formula of myths, Ojibwa, substance.]

Nuevas consideraciones sobre los algonquinos y el totemismo. La reciente publicación de un artículo que critica, de manera por lo menos rara, el esquema propuesto por primera vez en El Totemismo en la actualidad, ofrece una oportunidad de volver a la famosa tabla de permutaciones elaborada por Lévi-Strauss hace varios años. El presente texto muestra que las cuatro combinaciones posibles están efectivamente presentes en el área algonquina. Valiéndose de un grupo de Klein y de la fórmula canónica de los

* EHESS et musée du quai Branly, Paris [emmanuel.desveaux@ehess.fr].

Journal de la Société des Américanistes, 2004, 90-1, pp. 7-24. CSociété des Américanistes. 
mitos, se desarrolla la idea que el sistema " totémico » algonquino está completo. El fundamento semántico de tales operaciones resulta ser la denegación de la sustancia. De ahí siguen varias consideraciones relativas a las escalas regional y continental. [PALABRAS ClAVES : totemismo, Lévi-Strauss, grupo de Klein, fórmula canónica de los mitos, indios ojibwa, sustancia.]

Dans un article récent, publié dans un recueil d'hommages dédié à William C. Sturtevant ${ }^{1}$, Raymond Fogelson et Robert Brightman (2002) s'adonnent à une attaque en règle des thèses proposées il y a quarante-cinq ans par Lévi-Strauss dans Le Totémisme aujourd'hui et La Pensée sauvage, sur fond d'un conflit supposé entre écoles américaine et européenne. Dans un tel contexte, ils font de Frazer leur cheval de bataille : "It is tempting to see in Frazer's counter-positions American individualism matched against European social apriorists, but we won't pursue this here » (ibid., p. 309).

Partisans de réhabiliter l'interprétation substantialiste du totémisme qui avait cours au début du siècle dernier ${ }^{2}$, ils développent leur argument en trois temps qu'ils légitiment globalement comme une opération de rapatriement de la problématique sur ses terres d'origine, à savoir l'Amérique du Nord ou plus exactement son domaine algonquin. Après un retour au texte princeps, celui où apparaît pour la première fois le mot « totémisme », Fogelson et Brightman se placent sur le terrain de la philologie pour déboucher sur l'ethnographie d'un groupe donné, malheureusement en position marginale par rapport aux axes de discussion qu'ils ont choisis de suivre.

John Long, trafiquant anglais en fourrures installé à la fin du XvIII ${ }^{\mathrm{e}}$ siècle chez les Ojibwa, forge le mot «totémisme » (en réalité dotemism), à partir du mot dotem appartenant à la langue locale. La tradition anthropologique fait référence au texte de Long en soulignant habituellement que son auteur avait confondu l'appartenance à un clan spécifique qui possède une appellation animale, à savoir le "totémisme collectif», avec l'esprit tutélaire, en général lui aussi animal, acquis au cours de la quête de vision, laquelle est, par nature si l'on peut dire, individuelle. En réalité, selon Fogelson et Brightman, il n'y a pas vraiment confusion sous la plume de Long, car le principe d'organisation sociale de type clanique et le principe religieux de la tutelle spirituelle reposent sur la même chose. Leur démonstration emprunte un chemin tortueux qui finit par se mordre la queue puisque les principes de l'organisation sociale des Ojibwa « classiques » servent de prémisses et de conclusion à leur argument. Ils postulent en effet que le terme totem renvoie à la parenté, laquelle, pour eux, ne saurait être autre que celle de consanguinité patrilinéaire, puisque tel est le type d'organisation sociale qui prévaut chez les Ojibwa dont parlait Long. Ensuite, faisant appel à diverses autorités linguistiques (il ne s'agit en réalité que d'Ives Goddard, d'après une communication personnelle citée par Callender), ils nous instruisent du fait que 
l'étymologie du mot se trouve dans le radical verbal *o-te dont le champ lexical recouvre le thème de "vie en voisinage », voire de village. Il suffít donc de rapprocher la notion de cohabitation avec celle de patrilignage, d'affirmer que les Ojibwa dont on parle possèdent bien des clans avec animal éponyme, et l'on consolide l'idée classique (au sens pré-lévi-straussien) de totémisme clanique. Aucune importance que les clans ojibwa soient tout sauf des unités résidentielles (mais l'argument d'étymologie ne s'use, comme chacun sait, que lorsqu'on s'en sert) ! Du reste, Fogelson et Brightman remarquent que de nombreuses sociétés algonquines possèdent le mot totem ou des termes manifestement apparentés tout en ignorant les clans patrilinéaires. Ils auraient pu citer en exemple les Ojibwa septentrionaux, du moins ceux de Big Trout Lake chez lesquels nous avons fait notre terrain, qui, effectivement, utilisent le mot nidotem. Il signifie pour eux mon ami ( $n i$ : possessif). Il est clair que l'amitié s'entretient d'autant mieux que l'on se côtoie, donc que l'on co-réside, ne serait-ce que pendant le laps de temps où la relation en question s'éprouve réellement, notamment par des situations d'interlocution. Ce facteur de co-résidence dans l'établissement ou le renforcement de l'amitié prend d'autant plus de relief que nous sommes dans des sociétés qui, régies par des alternances saisonnières de concentration et de dispersion, se placent pourtant plutôt sous le signe de la dispersion, de l'éclatement des humains à travers d'immenses territoires. Jusqu'à un certain point, le rapprochement entre les uns et les autres y relève plus de l'exception que de la norme. Tout cela pour dire que si l'on suit le raisonnement étymologique - ce qui peut éventuellement se révéler éclairant -, c'est bien le critère de co-résidence qui apparaît comme le plus signifiant. Celui-ci, selon les groupes ou les dialectes, rencontrera une acception en termes de parenté ou en termes d'amitié. Mais cette notion de co-résidence est susceptible de s'appliquer aussi bien, peut-être, aux humains qu'aux entités extra-humaines, au premier rang desquelles les animaux.

Nul doute que Long fut un ethnographe honnête et qu'il fut, pour son époque, cultivé puisqu'il fait, ainsi que nous le rappellent Fogelson et Brightman par le biais d'une longue citation, le parallèle entre les croyances des Indiens et celles de Samuel Bernard, banquier juif de Louis XV. Cela étant, rendons-nous à l'évidence que l'enquête ethnographique, telle que nous la concevons à l'heure actuelle, n'était pas l'occupation principale de Long, ni probablement sa préoccupation principale. Celle-ci restait de saouler les Indiens afin de leur extorquer des fourrures au meilleur prix, ainsi qu'il le raconte complaisamment des pages et des pages durant (quitte à relire un texte fondateur, autant le lire en entier !). On doit alors admettre la possibilité qu'il se soit mépris sur les relations complexes que ses clients autochtones entretenaient avec les espèces animales et les esprits tutélaires. Remarquons que ladite quête de vision s'avère tout aussi compatible que l'hypothèse sociologique avec l'étymologie du mot totem entendu en termes de co-résidence. Il faut jeûner, supplier les esprits animaux, se lier d'amitié avec eux, là où ils demeurent. Les sites de jeûne sont précisément choisis par les 
dépositaires de la mémoire du territoire en fonction de cette exigence, ainsi que nous l'avions montré naguère (Désveaux 1988, pp. 285-288). Nous venons de le voir, les Ojibwa septentrionaux n'emploient pas le mot dotem pour désigner l'esprit tutélaire ; ils lui préfèrent l'expression opawakan (dont, paradoxalement, l'étymologie semble relever de la notion de vision et se rattache vraisemblablement à l'importante composante cree de leur vocabulaire). Mais cela ne serait pas une aberration en soi. Il est clair que, dans la langue ojibwa, il existe des variations dialectales et idiosyncrasiques ouvrant la possibilité à des usages différentiels du terme totem au sein des groupes ojibwa de la rive nord des Grands Lacs. Cette situation complexe et fluctuante prête pour le moins à confusion.

Mais si Fogelson et Brightman écartent l'idée d'une certaine confusion chez Long - non pas au sujet de la signification du mot totem, mais à celui d'une réalité ethnographique sophistiquée -, c'est parce qu'ils refusent de reconnaître à LéviStrauss le mérite d'avoir distingué les deux choses pour montrer ensuite qu'elles relèvent, à un niveau plus général et plus abstrait, mais à ce niveau seulement, de la même logique mentale : elles ne sont jamais que deux moments d'une combinatoire plus vaste qui se présente comme une table de permutation à deux fois deux entrées, mettant en rapport des entités naturelles et culturelles (espèce/groupe ; individu/personne) et engendrant quatre catégories de phénomènes observables dans l'ethnographie. Instrumentalisant Frazer qui avait dressé des cartes de distribution du totémisme «collectif » et « individuel » et avait constaté leur recoupement, Fogelson et Brightman induisent que ces deux formes se superposent, se confondent, en bref s'équivalent largement. Lévi-Strauss, lui, construit un champ de phénomènes distincts, mais liés entre eux par transformation. La démarche présente le double avantage d'être fidèle à l'ethnographie et d'offrir à l'interprétation un premier cadre analytique puissant (la propension humaine à catégoriser à partir du spectacle de la diversité naturelle). Nous sommes le premier à penser qu'un tel cadre n'épuise pas la question, mais nous persistons à croire que c'est l'une des plus sûres portes d'entrée pour comprendre les phénomènes dits totémiques ${ }^{3}$; nous allons y revenir.

Ainsi que l'on pouvait s'y attendre, le rejet de la leçon lévi-straussienne conduit Fogelson et Brightman au désastre intellectuel. Dans la section terminale de leur article, ils découvrent l'origine du totémisme chez les Penobscot, une " tribu » dont le territoire recouvrait le nord de la Nouvelle-Angleterre. L'ethnographie de référence de ces Algonquins, due pour l'essentiel à Speck et datant du début du $\mathrm{xx}^{\mathrm{e}}$ siècle, décrit la situation qui était la leur durant la seconde moitié du $\mathrm{XIX}^{\mathrm{e}}$ siècle, soit au terme, déjà, de deux siècles de contacts et de colonisation. Ce groupe dont l'organisation sociale était dépourvue de groupes de descendance patrilinéaire était divisé en une bonne dizaine de « bandes », c'est-à-dire de larges unités résidentielles. Les Penobscot n'avaient pas d'esprits tutélaires animaux, par essence, immatériels. Chaque individu possédait en revanche une ou plusieurs " amulettes-fétiches», autrement dit des objets personnels, lesquels, comme 
d'ailleurs les esprits tutélaires ojibwa, étaient susceptibles d'entrer en jeu dans les manipulations chamaniques. Le mot totem existe dans la langue ; il désigne l'ami, mais également, l'affin. Mais tout cela n'empêche pas les Penobscot de posséder un authentique système totémique qui se donnerait sous un mode d'appellation des bandes lié à des espèces animales. En effet, les différentes bandes penobscot s'appelaient en fonction de la ressource animale la plus densément présente dans la section du territoire global qui lui revenait : les « lièvres » là où il y a le plus de lièvres, les " castors » là où ces rongeurs pullulent, les " élans » là où l'espace exploité abonde en élans, etc. Il convient d'en conclure, selon Fogelson et Brightman, que le totémisme procède d'une adéquation entre une espèce animale et un groupe par le truchement du régime alimentaire privilégié qu'imposerait telle ou telle section du territoire. Et les auteurs, pour composer bonne figure, de faire remarquer que, parfois chez les Ojibwa, il existe la possibilité de consommer la chair de l'espèce éponyme. Certes, mais cela reste l'exception et non la règle, cela arrive seulement dans certaines circonstances, notamment en cas de pénurie. Enfin, pour couronner leur démonstration, les auteurs s'adonnent à une sorte d'emballée structuraliste (ou ce qu'ils pensent en être) : les Penobscot occuperaient ainsi le pôle diamétralement opposé à celui des aborigènes australiens et de leur soi-disant totémisme, corollaire de l'interdiction stricte faite au chasseur de manger l'animal qu'il a tué. Cette ouverture maximale de la discussion se révèle pour le moins inattendue puisque l'argument, balisé au début par la philologie, s'était jusqu'alors cantonné à l'aire algonquine. D'autant que ces auteurs déclaraient en préambule de façon péremptoire qu'ils allaient justement considérer le phénomène totémique sur sa terre d'origine, à savoir l'Amérique du Nord. Mais, une fois de plus, nous ne sommes pas à nous arrêter à une contradiction supplémentaire chez nos auteurs.

À dire vrai, les objections abondent désormais à l'esprit du lecteur qui serait parvenu à ce stade de la démonstration. À partir du moment où l'on choisit d'introduire les Penobscot dans la discussion, pourquoi ignorer dans ce cas la signification qu'ils donnent eux-mêmes au mot totem après avoir prêché les vertus heuristiques de la philologie. Il nous semble en effet qu'une inversion d'une acception consanguine, puisque patrilinéaire, du terme à une acception le rangeant du côté de l'affinité aurait mérité un commentaire. Enfin, si l'on décrète que les Penobscot offrent la clé de compréhension du totémisme en vigueur chez les Ojibwa, malgré l'absence ou la présence de clans et d'esprits tutélaires, malgré le fait qu'ici on mange son éponyme et que là on évite de le faire, on s'interroge sur la relation des premiers aux seconds. Est-elle de nature historique ? On ne voit pas comment il pourrait en être autrement : il n'existe aucune autre connexion imaginable dans le schéma proposé par Fogelson et Brightman. Les Ojibwa étaient-ils à l'origine des Penobscot qui, en migrant, auraient adopté le principe du patrilignage, mais auraient conservé leur « emblème» alimentaire, pour reprendre les mots de Durkheim, sans s'interdire d'en manger, inspirés sans doute 
par la nostalgie de la terre natale ? Symétriquement, pourquoi les Penobscot n'auraient rien changé pendant des siècles à leurs institutions? Si les deux théoriciens se gardent bien d'assumer comme telles ces spéculations diachroniques, pourtant implicites dans leur propos, c'est parce qu'ils savent que ce genre de raisonnement a déjà été réfuté maintes et maintes fois, et ce depuis plus d'un siècle. Mais de surcroît, quid de ces formes de totémisme en Amérique du Nord qui ne sont pas algonquines? Notons à ce propos qu'être appelé par sa principale nourriture n'a rien d'exceptionnel. Sans aller chercher le cas, classique en Amérique du Nord, des Païutes (Fowler 1982), on signalera celui des Indiens de Big Trout Lake. À l'instar des Penobscot, ces Ojibwa septentrionaux n'avaient pas d'organisation en clans patrilinéaires, mais ils partageaient avec la plupart des groupes ojibwa le complexe de la tutelle spirituelle. Ils étaient trivialement désignés par leurs voisins méridionaux comme « mangeurs de poissons ». Effectivement, les espaces qu'ils exploitaient dans l'intérieur de l'extrême nord-ouest de l'Ontario s'avéraient, toutes proportions gardées compte tenu des latitudes où ils se trouvaient, relativement riches en ressources halieutiques. Mais que dire maintenant de ces mêmes voisins, notamment ceux sédentarisés aujourd'hui à proximité du lac Weagamow et qui sont affublés du nom de odidjakoosuk, " petites grues »? Ce sobriquet collectif se justifie, pour les gens de Big Trout Lake, par un accent qui évoque le chant rauque de la jeune grue à leurs oreilles. Ces deux appellations sont totémiques, si l'on suit Fogelson et Brightman, l'une d'ordre primaire et l'autre dérivée, bien qu'un régime alimentaire fondé sur la chair de grue semble un peu austère ! Plus sérieusement, on notera que la première relève plutôt de la métonymie et la seconde de la métaphore, mais que, dans les deux cas, elles apparaissent « rationnellement » motivées. Est-ce parce que ces appellations émanent de l'extérieur ? ${ }^{4}$ Lévi-Strauss avait déjà soulevé la question dans La Pensée sauvage, sous l'angle de leurs fondements en nature des classifications, mais pas sous celui d'une éventuelle distinction à opérer entre désignations internes et externes. Voilà une piste à poursuivre plutôt que de tenter; comme le font Fogelson et Brigthman, de faire revivre à toute force des paradigmes dépassés depuis des lustres.

Toutefois ce n'est pas cette piste que nous aimerions emprunter pour l'instant. Car une autre, plus prometteuse encore, s'ouvre à nous grâce au remarquable article de William L. Merrill (2002) qui, à quelques pages d'intervalle, suit celui que nous venons de longuement discuter. Ce texte traite de l'étrange croyance qu'ont les Rarámuri - autodénomination des Tarahumaras - en des métamorphoses animales. Ces Indiens des montagnes du Mexique septentrional s'avèrent être, à l'instar de la plupart des populations traditionnelles, de fins observateurs de la nature. Leur savoir en matière d'éthologie animale est très étendu et recouvre globalement celui des zoologues patentés. Pourtant, ils croient que certaines espèces animales, en nombre restreint, sont susceptibles de compter; dans leur rang, des individus capables de se métamorphoser en individus appartenant à une 
autre espèce. Ainsi plusieurs petits rongeurs peuvent devenir des chauve-souris ; des poissons, en particulier ceux de type poissons-chats, prolongent leur existence sous forme de loutres; des écureuils arboricoles se métamorphosent en boas constricteurs, une autre espèce d'écureuils coutumiers des rochers, en serpents à sonnette. Enfin, les chèvres, les cochons et les chats - animaux domestiques d'origine européenne - deviennent respectivement des chevreuils, des pécaris et des ocelots une fois échappés à la surveillance des humains et supposés les avoir fuis afin de retourner à l'état sauvage.

En réalité, il s'agit là du noyau d'un corpus de croyances, plus ou moins étendu selon les informateurs, qui admet la possibilité de la métamorphose interspécifique. Les humains sont d'ailleurs concernés. Les Rarámuri pensent possible en effet qu'un humain se transforme après sa mort en animal, se greffant ainsi au complexe typiquement mésoaméricain du nahualisme. Pourtant ces croyances détonnent surtout dans le contexte américain où, en général, on a l'impression que les cultures font un pari presque pascalien sur la stabilité des espèces animales. C'est là que la théorie des ontologies reprise par les cognitivistes trouve sa justification mais c'est également là un des grands fondements du totémisme : parce que la différence entre un ours et un castor est perçue comme intangible, elle inscrit l'écart, décrété tel, entre ceux qui appartiennent au clan de l'ours et ceux qui appartiennent à celui du castor. Le caractère exceptionnel de ces croyances en une éventuelle métamorphose d'une espèce animale en une autre n'échappe pas à Merrill. Cherchant à constituer une base comparative afin de mieux interpréter le phénomène, il ne mentionne pas d'autres exemples américains et se tourne plutôt vers deux populations des hautes terres de Nouvelle-Guinée, les Kalam et les Rofaifo, et un groupe indonésien, les Nuaulu de l'île de Saram. Il montre qu'au-delà d'une opération semblable de notre point de vue, la métamorphose trans-spécifique, les Rarámuri se distinguent des trois autres exemples répertoriés. Pour ces derniers, la métamorphose relève d'un principe taxinomique annexe. Il s'agit de rendre compte de la proximité, hiérarchisée, d'espèces animales à la morphologie proche. Il s'agirait, en schématisant à l'extrême, de rapporter une forme animale mineure (en taille ou en quantité) à des formes plus importantes au regard de l'ethnozoologie locale. Un rat de brousse devient un rat géant, un opossum à queue rayée devient un cuscus tacheté, qui est lui aussi un marsupial mais de taille sensiblement supérieure, etc. Le fait que ces métamorphoses soient consignées dans la mythologie confirme l'analyse en termes d'ajustement taxinomique. Il semble que la situation soit différente dans le cas rarámuri. Mis à part les petits rongeurs qui deviennent des chauve-souris, métamorphose presque convenue dès lors que le principe en est accepté, les transformations rarámuri concernent des espèces qui partagent le même habitat. D'ailleurs, précisément, les informateurs racontent que l'écureuil des rochers entre dans une anfractuosité du terrain, se dépouille de sa nature d'écureuil à l'abri des regards de tous pour endosser celle, désormais visible par tous, de serpent à sonnette. Merrill signale 
que ses informateurs n'ont jamais vu la métamorphose s'effectuer. Adeptes d'une philosophie perspectiviste dont l'anthropologie contemporaine commence à vraiment prendre la mesure, d'une extrémité à l'autre du Nouveau Monde ${ }^{5}$, ils soutiennent au contraire que les animaux concernés se cachent délibérément afin de changer d'apparence. Dans ces circonstances, ils fuient plus encore que d'habitude le regard des humains, comme s'ils étaient parfaitement conscients du caractère choquant pour notre perception catégorielle de la transmigration d'un individu d'une espèce animale vers une autre.

Distinguant nettement, ainsi que nous l'avons vu, le modèle des métamorphoses rapporté par les autochtones de la Sierra Madre de ceux répertoriés dans le Pacifique, Merrill semble y voir l'expression, dans le registre zoologique, de l'un des fondements de la pensée rarámuri. Celle-ci en effet conçoit le temps comme une succession de mondes. La métamorphose des animaux offre une image toujours renouvelée de cette éventualité de mutation absolue du monde au moment du passage d'un âge à un autre. En serait l'indicateur les dires de ses informateurs selon lesquels c'est à un âge avancé que se manifeste cette propension au changement chez certains sujets animaux. Dans les pensées mésoaméricaines, on le sait, ce sont des mondes anciens, usés par les cycles saisonniers qui se disloquent pour donner naissance à des âges nouveaux. Le corps biologique, ses rythmes et les échéances qui l'attendent, deviennent alors le miroir de l'épisodique épuisement cosmique. Le nahualisme trouve ici sa place, tout comme l'esthétique, en définitive, du plateau central. De pierre ou de céramique, la statuaire aztèque excelle à rendre sensible, donc crédible, la métamorphose ${ }^{6}$. L'interprétation que Merrill propose de l'attitude des Rarámuri face à leurs animaux domestiques d'origine européenne renforce l'analyse. Si, d'un côté, ces Indiens les ont complètement intégrés dans leur économie et dans leur univers mental, d'un autre côté, ils leur reconnaissent une propension particulière à la transformation. Or cette dernière se traduit par leur réincarnation en des espèces animales qui apparaissent d'un point de vue morphologique comme leurs équivalents sauvages, donc préhispaniques. L'anthropologue décèle dans cette série de transformations relative aux animaux européens une sorte de symptôme régressif où l'amnésie d'une introduction historiquement datée s'accompagne de la vivacité d'une croyance en des mécanismes de métamorphose zoologique directement associés à l'état antérieur.

Sans vouloir invalider, au contraire, cette interprétation audacieuse et séduisante, nous aimerions revenir sur deux points dont Merrill a peut-être sous-estimé les implications. Cela devrait nous permettre de dépasser sa perspective strictement culturaliste - même s'il embrasse toute l'aire nord-mexicaine - et de rejoindre la question du totémisme dans l'optique d'un comparatisme panaméricaniste. Le premier est le suivant : seuls quelques individus d'une espèce donnée choisissent, ou possèdent la capacité, de se transformer en représentants d'une autre espèce. Tous les boas ne sont pas des écureuils réincarnés. La majorité d'entre eux 
naissent, croissent et meurent dans leur être de boas tout au long de leur existence. Second point : nous l'avons vu précédemment, la métamorphose intervient toujours à un âge avancé ; en d'autres termes, elle se présente, pour ceux qui en ont les moyens, comme une alternative à la mort pure et simple de l'individu. Ces métamorphoses sont plutôt considérées d'une façon négative. Les espèces de destination (serpents, loutre, chauve-souris) sont synonymes de danger soit pour les humains, soit surtout pour les espèces susceptibles de se transformer en elles, ce qui leur donne globalement une connotation cannibale : la loutre mange des poissons, les serpents se nourrissent des mammifères qui partagent leur habitat, etc. Ces métamorphoses signalent des individus qui refusent l'échéance normale de la mort en se faisant prédateur d'un ordre supérieur, aux dépens pour l'essentiel de leurs propres congénères.

Nous sommes de ceux qui pensent que Le Totémisme aujourd'hui reste l'une des pierres angulaires de toute discussion touchant aux relations entre les animaux et les hommes. Pourtant, lorsque nous le lisions pour la première fois, nous avions éprouvé un certain malaise qui ne s'est jamais dissipé depuis. Le passage scabreux à nos yeux se situe au début du premier chapitre, là où, après avoir introduit sa table des permutations, Lévi-Strauss soutient son hypothèse en fournissant des exemples ethnographiques correspondant à chacune des combinaisons énoncées. Pour les deux premières, ils furent faciles à trouver puisque on les rencontre dès le texte de Long. Et même si le clan totémique et l'esprit tutélaire y sont quelque peu confondus, toutes les enquêtes ethnographiques menées depuis le XVIII ${ }^{\mathrm{e}}$ siècle chez les Ojibwa confirment bien l'existence des deux institutions. En revanche, l'exercice se complique un peu lorsqu'il s'agit d'illustrer les deux combinaisons suivantes, à savoir individu (animal)/personne et individu/groupe (humain). Dans un cas, Lévi-Strauss (1962, p. 24) a recours à l'ethnographie des Mota, dans les îles Banks en Océanie, puis enchaîne : "On peut ajouter l'exemple de certaines tribus du groupe Algonquin, qui supposent qu'une relation particulière s'établit entre le nouveau-né et tel animal qu'on aura vu s'approcher de la cabane familiale $»$.

Reconnaissons que, dans ces pages cruciales du Totémisme aujourd'hui, les renvois bibliographiques s'avèrent être plutôt déficients, décourageant ainsi le lecteur de retourner à la source. Cependant la validité de l'information ne fait guère de doute pour nous, dans la mesure où, chez les Cree de la baie James au Nord, donc à la marge de la zone algonquine centrale dont parle manifestement Lévi-Strauss, l'ethnographie nous offre l'inversion parfaite de cette coutume. À la mort du père, le fils doit veiller sur sa tombe. La nuit, un animal - lièvre, hermine, marte, etc. - est censé s'approcher, voire marcher sur celle-ci. Cet animal singulier est le messager de l'esprit du défunt. Seule sa présence en ces lieux permet au fils de revendiquer l'héritage spirituel de son père (Tanner 1979, pp. 130-131). Mais c'est la dernière combinaison, celle qui associe un individu animal avec un groupe humain, qui fut peut-être la plus acrobatique à illustrer. Lévi-Strauss nous incite 
à la voir dans les « animaux sacrés » qui feraient l'objet d'une protection collective en Australie ou en Afrique, ou encore dans le culte des ongon, ces images d'animaux qui font l'objet d'un culte en Sibérie. On pourrait discuter la pertinence de ces exemples. Les animaux "sacrés » retenus en captivité pour des besoins cultuels sont rarement uniques, en outre les situations de ce type se révèlent extrêmement hétérogènes. Dans ces conditions, comment être certain qu'ils ne représentent pas une espèce dans son ensemble ? Quant au culte des ongon, il repose sur des images. Là encore, il paraît difficile de s'assurer que celles-ci représentent bien un animal singulier et non un prototype de l'espèce. Ou encore que la singularité qui leur est prêtée est celle du représenté et non pas de la représentation. Mais, au fond, ce qui nous perturbait le plus dans le choix de ces illustrations ethnographiques tenait à la série interrompue des exemples algonquins. Si les quatre combinaisons procèdent d'une même logique mentale, comment se fait-il que trois d'entre elles trouvent à s'exprimer dans des matériaux algonquins, donc au sein d'une aire culturelle relativement restreinte, et que l'une en soit exclue ?

Or il n'en est rien. L'ethnographie de l'aire concernée recèle bien la combinaison individu animal/groupe humain, ainsi que nous allons le découvrir un peu plus loin. Mais, avant cela, en quoi les croyances rarámuri nous aident à mieux définir les enjeux de cette combinaison ? Lévi-Strauss pose - ou postule selon le point de vue - la différence entre le collectif et l'individuel, que cela concerne la série naturelle, les animaux, ou culturelle, les hommes. Il n'explore pas pour autant les implications de cette différence. Or les Rarámuri, par le biais de la métamorphose interspécifique comme palliatif de l'absence de destin eschatologique, nous apprennent qu'une aftīnité particulière lie la notion d'individu (ou de personne) et la mort. Ce sont seulement quelques écureuils des rochers qui deviennent des serpents à sonnettes et non pas tous. On l'aura compris : il s'agit pour ces privilégiés d'échapper, au moins pour cette fois, à la mort biologique, synonyme, semble-t-il dans leur cas, d'un destin eschatologique assez terne. Plus que la naissance, car celle-ci n'est jamais qu'un sortir de l'indétermination ${ }^{7}$, qu'une mise en place d'une potentialité, la mort confirme la nature singulière de chaque être vivant. Une vie qui s'achève, c'est une succession unique de faits et gestes, de paroles et de non-dits, de succès et d'échecs qui s'interrompt ; c'est la forclusion d'une existence, dans ce qu'elle a de foncièrement irréductible. Le profond sentiment de perte qu'elle inspire s'explique par cette singularité absolue ${ }^{8}$. La mort est donc l'instance d'aflirmation la plus forte de l'individualité. En ce sens, elle s'oppose à l'ontologie des espèces animales qui se fonde, on le sait désormais, sur une intuition forte quant à sa stabilité - un loup sera toujours un loup, un castor, un castor - et, ce qu'on souligne moins couramment, sur une phénoménologie de la naissance - une louve engendrera toujours des louveteaux, une femelle castor des petits castors, etc.

Le détour par les Rarámuri nous a conduit à reconnaître la pertinence de la mort pour le système totémique. Revenons à présent sur notre quête de la 
combinaison individu animal/groupe humain. Lorsque nous étions sur le terrain il y a une vingtaine d'années à Big Trout Lake, nous avions recueilli de nos plus anciens interlocuteurs des informations relatives à deux pratiques rituelles qu'ils n'avaient eux-mêmes pas connues mais dont ils avaient entendu leurs aînés parler. Leurs descriptions, bien que sommaires, étaient d'une grande cohérence. L'une de ces pratiques se donne, en apparence, comme un rituel d'initiation. Lorsqu'un enfant ou un jeune adolescent tuait, pour la première fois de son existence, un type de gibier et qu'il inaugurait sa relation de prédation vis-à-vis d'une espèce animale, les adultes qui l'accompagnaient, autrement dit qui l'avaient conduit vers cette proie, devaient partager avec lui un repas au cours duquel la carcasse entière de l'animal devait être mangée. Il s'agit en général d'hommes et le repas se déroulait en forêt, à l'écart des campements familiaux et donc des femmes. La seconde pratique repose sur le même principe de dévoration entière d'une carcasse animale. Elle relève du complexe du festin sans fin, qui est par ailleurs un motif très commun dans toutes les mythologies du subarctique algonquin. Elle évoque aussi des festins de viande de caribous chez les Naskapi. Un chasseur a tué un caribou. Il invite ses amis, un groupe d'hommes, à venir faire cuire et à manger entièrement la carcasse de l'animal, à l'exclusion des os et des tendons. Cuisson et manducation intégrale doivent intervenir entre le coucher et le lever du soleil. Il est clair que les convives de cet étrange repas mangeaient au-delà de la satisfaction de leurs besoins physiologiques, au-delà également de celle des plaisirs gastriques. Nous avions montré, à travers notre analyse de la figure du démiurge Jakabish, qu'une conjonction sémantique forte prévalait entre l'alternance du jour et de la nuit et la masculinité ${ }^{9}$. Elle se confirme ici dans ce rituel nocturne qui possède également de façon manifeste une connotation confrérique. Mes informateurs me précisèrent que, s'il restait de la viande au petit matin, ces convives s'exposaient aux pires calamités. En revanche, s'ils parvenaient à leur dessein de tout manger, ils renforçaient mutuellement leur puissance " chamanique » respective. C'est donc bien une relation de solidarité organique entre quelques hommes qu'instaure cette curieuse cérémonie directement liée à la mort d'un individu animal. En bref, cette forme rituelle, ainsi que la précédente qui intéresse un cercle de proches parents du chasseur impétrant, atteste bien l'existence de cette combinaison individu animal/groupe d'humains au sein de l'aire algonquine. Du coup, s'éclaire l'interdit de tuer ou de manger qui frappe l'animal éponyme du totémisme « clanique » des Ojibwa méridionaux et qui a fait couler tellement d'encre chez les théoriciens du totémisme depuis le $\mathrm{XIX}^{\mathrm{e}}$ siècle. Il y a tout simplement transformation au sens lévi-straussien, sous forme d'inversion, entre cet interdit permanent de manger un représentant de l'espèce et l'obligation de le faire en un laps de temps prescrit. Ou, pour dire les choses autrement, tuer et manger un représentant de l'espèce revient à le désigner comme individu. S'abstenir, symétriquement, d'un tel acte vis-à-vis de tous les individus d'une espèce revient à lui conférer dans sa totalité une valeur représentative. 
Grande est pour nous la tentation d'élargir ce groupe de transformation binaire à un groupe à quatre éléments, de type groupe de Klein, afin d'intégrer les deux autres combinaisons, espèce/individu humain (« totémisme individuel classique ») et individu animal/individu humain, combinaisons dont nous savons que la réalisation ethnographique est attestée dans l'aire ojibwa ou sur ses marges. On sait que la combinaison espèce/individu humain joue un rôle déterminant chez les Ojibwa durant l'initiation. C'est à partir de ce moment que l'individu est supposé entrer dans l'âge adulte, autrement dit devenir une personne, dotée d'une grande capacité d'autonomie. Dans la lignée de Speck et de son atomisme social, Hallowell (1976, p. 386) avait très bien vu ce primat de l'individu chez les Ojibwa, notamment sur la société considérée comme lieu d'intérêts communs. Nous avons donc bien à faire à un acte de naissance spirituelle - et non biologique - de l'individu humain, que conditionne sous sa forme immatérielle une espèce animale. Par contraste, c'est bien un individu animal qui intervient au moment de la naissance ou de la mort biologique d'un individu humain. Les traces que sa présence physique a imprimées sur le sol ou dans la neige au moment de son passage soulignent sa réalité concrète. Nous pouvons dresser le groupe de Klein suivant :

\section{(x)totémisme de clan}

(1/x)repas chamanique

\section{(-x)totémisme individuel}

\section{$(-1 / x)$ traces dans la neige}

La ligne horizontale supérieure est celle de la virtualité de l'animal versus l'inférieure qui est celle de sa réalité. La première ligne verticale à gauche est celle d'une identité de groupe versus celle de l'individualité. La diagonale de (x) à $(-1 / x)$ est celle de la permanence versus de l'éphémère, tandis que celle qui relie $(-\mathrm{x})$ à $(1 / x)$ correspond à deux modes d'acquisition du pouvoir spirituel, l'un personnel et cumulatif, l'autre collectif et ponctuel. En réalité $(x)$ n'est pas tant une initiation qu'une inauguration. Tous les informateurs nous ont expliqué que le jeûne en quête d'opawakan, esprit tutélaire sous forme animale, ne s'endurait pas nécessairement qu'une seule fois dans l'existence. Au contraire, à tous les stades de la vie, l'individu avait la possibilité de retourner jeûner en forêt, soit pour renforcer la qualité de sa relation avec son esprit tutélaire que l'initiation adolescente lui a attribué, soit pour tenter d'en acquérir de nouveaux. Cette précision nous importe particulièrement car elle introduit dans notre schéma la pluralité des espèces (versus l'unicité de l'animal effectivement mangé). Notons enfin que notre groupe de Klein reflète aussi avec eflicacité la dimension publique ou secrète des diverses relations entre les hommes et les animaux. L'affiliation d'une personne à un clan est parfaitement connue de tous comme l'est son espèce éponyme. En revanche, l'esprit tutélaire de chacun doit demeurer secret. Mes interlocuteurs ont toujours insisté sur ce point. Quant au repas chamanique, 
il s'inscrit dans le cadre d'un secret partagé. Il se déroulait à l'écart du campement, à la dérobée du regard des femmes et de tous ceux qui en étaient exclus. Enfin, les traces laissées sur le sol ou la présence furtive d'un animal donné relève dans les faits d'une sorte de surcapacité d'observation ou de surveillance. Retenons de nouveau l'option perspectiviste. Malgré la vocation de la présence animale à rester cachée - à l'exception des oiseaux, les animaux tentent toujours de se dérober à la vue des hommes -, quelqu'un, parmi les humains, a réussi à la détecter ou du moins à en déchiffrer les traces, elles-mêmes au demeurant de nature éphémère.

Existe-t-il une formule canonique des mythes (dont, selon nous, la pertinence dépasse le cadre de la mythologie stricto sensu) dérivé de ce quadrant ${ }^{10}$ Il semble que oui. Elle se présente très simplement ainsi :

$$
\begin{aligned}
& \mathrm{F}_{\text {être }} \text { (humain) : } \mathrm{F}_{\text {classer }} \text { (animal) : : } \\
& \quad \mathrm{F}_{\text {classer }} \text { (humain) }: \mathrm{F}_{\text {animal }} \text { (non-être) }
\end{aligned}
$$

Autrement dit, la relation entre l'existence des humains et les catégories distinctes d'animaux - les espèces - est équivalente à la relation entre la classification des humains (en clans, en enfant ou en adulte par l'initiation, en groupes de type confrérique, en tant qu'humain et non-animal, car c'est au fond de cela qu'il s'agit dans l'affaire des traces laissées dans la neige) et la propension des animaux à échapper à leur condition de substance (non-être). Expliquons ce dernier point. On ne peut qu'être frappé par le fait que toutes les modalités de totémisme algonquin que nous avons vues tendent vers un but : la négation de la substance. Cela est clair dans le cas des totémismes classiques collectif et individuel, qui manipulent exclusivement des réalités mentales, mais cela se révèle également vrai dans celui des deux autres combinaisons du quadrant. Le festin chamanique a pour effet de faire disparaître, de dissoudre, d'anéantir le plus rapidement possible la chair du caribou partagée par les convives, tandis que les traces laissées dans la neige traduisent ce que l'on pourrait appeler en hommage à Louis Marin une présence-absence, en tout état de cause une présence évasive. Seule la perception visuelle l'entérine, au détriment de tout autre sens, et notamment de celui du toucher, moyen privilégié d'apprécier pleinement la substantialité d'un être ou d'une chose.

Le système dans son ensemble équivaut en conséquence à une pétition de principe en faveur d'une ontologie animale immatérielle, littéralement désincarnée ${ }^{11}$; il revient en somme à un dispositif de négation de la substance. Notre propre opération de "ré-algonquinisation » du totémisme débouche sur une conclusion qui se situe à des années-lumière de celle de Fogelson et Brightman qui voyaient au contraire dans la substance à consommer l'explication - historique de l'institution. Notre résultat possède d'inévitables corrélats que nous aimerions brièvement évoquer pour finir. 
Lors de nos séjours à Big Trout Lake, dans les communautés environnantes ou dans les camps de trappe, nous avions toujours été intrigué par l'absence de tout objet autochtone à valeur ou à signification rituelle. Certes, l'acculturation pouvait tenir lieu d'explication, mais jusqu'à un certain point seulement, car, pour le reste, nos hôtes parlaient encore leur langue et tout, dans leur comportement, notamment social, attestait de la vitalité de leur culture d'origine. Aujourd'hui, nous pouvons légitimement nous demander si ce défaut de matérialité n'est pas plutôt à mettre en rapport avec leur système " totémique» de croyances et le déni de la substance qu'il implique. Dans le même ordre d'idée, on se demandera si le principe même du midédewin, confrérie secrète des Ojibwa des pourtours des Grands Lacs, n'est pas rendu possible par le déni de substance. Il s'agit ici, en effet, de « tuer » puis de « ressusciter » rituellement les membres de la confrérie à chacune des accessions aux quatre grades qu'elle comporte. Ce déni contribue à contrecarrer le caractère contre-intuitif de cette croyance en une possibilité de résurrection, laquelle se retrouve présente de façon lancinante dans tout le « folklore » autochtone de la région. Il s'accorde parfaitement enfin avec une horreur de la putréfaction qu'énoncent à la fois la mythologie et les pratiques quotidiennes (Désveaux 1988, p. 218).

Ce déni dont le totémisme algonquin serait porteur repose donc la question de la distribution spatiale des institutions ou des « croyances ». On notera qu’à la périphérie de la zone sur laquelle nous avons concentré notre attention, surgit l'usage de la matérialité, là où précisément prévaut sa négation. Ainsi des amulettes individuelles dont usent les Penebscot, comme d'ailleurs leurs voisins Montagnais au nord, de l'autre côté du Golfe du Saint-Laurent. Ainsi également des autels portatifs ou des paquets cérémoniels (bundles en anglais) à valeur collective ou clanique que l'on rencontre chez les Fox et autres groupes du moyen Mississippi au sud ou encore parmi les tribus des Plaines. Ces amulettes ou le contenu de ces paquets sont en général des fragments de dépouilles animales : os, peaux desséchées, dents, griffes, têtes d'oiseau quasi-lyophilisées,... autrement dit, au statut ambigu par rapport à la notion de substance. Ils appartiennent sans conteste à l'ordre de la matérialité, mais à une matérialité minimale, embryonnaire, en tout état de cause non comestible. En bref, on pourrait parler d'une matérialité non substantielle. Si l'on admet que la substance se définit comme une matérialité pleine, saturée, nourrissante, féconde, la matérialité de ces objets marque une ébauche de sortie du déni de substance.

Dans sa très belle monographie sur les Guayaki, Pierre Clastres (1972, pp. 252-254) nous dit que si un homme donne de la viande à manger à une femme enceinte, il devient un sorte de père supplétif de l'enfant à naître. Cette reconnaissance en paternité secondaire, susceptible d'être partagée d'ailleurs, appartient de toute évidence au complexe de la co-paternité, très répandu en Amazonie. Toutefois elle le fait en jetant une lumière particulièrement crue sur le primat de la substance dans cette partie de l'Amérique. Nos collègues sud-américanistes n'ont 
de cesse depuis quelques années de souligner l'importance que revêt là-bas la prédation, entendue comme propension à l'ingestion, à la dévoration, à la consommation alimentaire. Cette prédation conditionne les relations interspécifiques et, très souvent, intraspécifiques, débouchant sur le cannibalisme. Ce complexe de la prédation est bel et bien pour eux synonyme d'une sorte de surdétermination, si l'on peut dire, de la consistance de la substance, mais précisément à l'intérieur des limites des corps qui, eux, revêtent l'apparence d'une espèce ${ }^{12}$. On voit s'esquisser un large système de transformation dont les pôles résident, d'une part, dans le totémisme algonquin négateur de substance, dans cette hypertrophie sud-américaine de la substance, d'autre part. Le thème, qui surgit en Mésoamérique, de la métamorphose d'une espèce animale en une autre ou d'un humain en un animal participe à ce système de transformation : il en représente un terme moyen en imaginant une possibilité de transmigration, limitée à certaines circonstances particulières, de la substance d'un corps animal dans un autre. La métamorphose rarámuri instruit la synthèse entre les deux pôles par amoindrissement de leurs prérogatives respectives. Si la substance y prévaut comme en Amérique du Sud, elle se voit subordonnée pourtant à des procédures qui entrent en contradiction violente avec sa vocation première de " remplir » de manière récurrente et stable des entités ontologiquement prédéfinies. Symétriquement, la métamorphose, au niveau de quelques espèces et quelques individus seulement - somme toute, de manière très partielle mais signifiante pour la totalité du système par la brèche qu'elle ouvre - opère un décloisonnement des catégories tenues pour rigoureusement hermétiques par la perception des animaux en général et par le totémisme algonquin en particulier.

Une dernière remarque : notre démarche de « ré-algonquinisation » du totémisme nous a conduit à apprécier une nouvelle fois la dimension américaniste de certains phénomènes, donc à poser la question éventuelle d'existence de mégaaires culturelles. De fait, nous avons restreint la portée du totémisme à une zone géographique donnée, ne serait-ce que pour montrer, pour suggérer plutôt, que ses répercussions s'étendaient bien au-delà, à l'échelle d'un continent entier. Nous sommes conscient que la démarche est à la fois dans la continuité et en rupture par rapport à celle de Lévi-Strauss. L'auteur du Totémisme aujourd'hui avait démantelé les dernières ruines d'un paradigme de l'anthropologie qui datait de la fin du $\mathrm{XIX}^{\mathrm{c}}$ siècle afin de reconstruire dessus un nouveau, celui d'une logique classificatoire fondée sur les données du sensible. Il le reconstruisait cependant presque à l'identique dans ses proportions, plaçant d'emblée la discussion à l'échelle de la planète entière. Il faisait ainsi l'économie d'une réflexion sur la notion d'aire culturelle - étroite ou élargie. Encore qu'il consacrait un long chapitre exclusivement à l'Australie. Or, on l'aura compris, ce qui se passe en Australie, sur les îles Banks ou aux Andamans, en Afrique également ${ }^{13}$, n'a probablement pas grand-chose à voir; selon nous, avec ce dont nous avons parlé jusqu'ici, sinon à un niveau très général - à savoir que le spectacle de la diversité 
naturelle, animale en particulier, inspire la pensée humaine qui, elle-même, se caractérise par une puissante faculté à la catégorisation. Dès lors que l'on envisage des rapprochements plus étroits entre éléments d'ethnographie provenant d'aires culturelles très distinctes, nous nous interrogeons sur leur pertinence. Lowie (1912, pp. 24-42) fut en son temps sensible au phénomène de «convergence " et à la cohorte de faux-semblants qu'il draine dans son sillage. Il nous mettait en garde contre la tentation d'expliquer de la même façon des faits ethnographiques qui se donnent à l'observateur comme très similaires. Reste à définir la bonne échelle d'appréhension et de comparaison première des phénomènes. Nul doute que, depuis Les Mythologiques de Lévi-Strauss, l'un des grands plaisirs de l'américaniste réside dans le fait que celle-ci s'impose d'elle-même, épousant d'emblée les contours du continent. Convenons que la facilité qui nous est offerte ici, loin de nous détourner de la question du comparatiste, nous confère une responsabilité particulière en la matière vis-à-vis de l'ensemble de la discipline. *

* Manuscrit reçu en février 2004, accepté pour publication en mars 2004.

\section{NOTES}

1. Voir le compte rendu de ce recueil dans ce même volume du Journal de la Société des Américanistes.

2. Saluons à ce propos la parution récente d'un ouvrage, celui de Frederico Rosa (2003), qui permet de mieux apprécier les multiples facettes de ce débat.

3. Voir le chapitre XVI (« Totem et phénomène ») de notre ouvrage Quadratura Americana. Essai d'anthropologie lévi-straussienne (2001).

4. Adomoawan kenoshe (litt. "ils mangent du poisson »). Entendons-nous bien, il s'agit de la formulation donnée, dans son propre parler, par notre informateur qui relate ainsi une expression qui aurait cours, selon lui, chez ses voisins pour le désigner lui et ses semblables. Contre toute attente - en faisant maintenant référence à une autre polémique fameuse impliquant Lévi-Strauss --, on dira que la dialectique sartrienne de l'autre comme miroir de soi peut se révéler utile dans la discussion sur le totémisme.

5. Voir le chapitre VII (« Perspectivismo e multinaturalismo na América indigena ») de l'ouvrage d'Eduardo Viveiros de Castro (2002).

6. Voir, si besoin était de s'en convaincre, l'impressionnante exposition présentée l'année dernière à Londres (Matos Moctezuma et Solis Olguin 2003).

7. Surtout dans les pensées amérindiennes qui, bien que très au fait du caractère sexué de la génération chez les animaux et les humains, ignorent l'hérédité biologique (Désveaux 2001, pp. 164168).

8. Nos analyses convergent avec celles de Pascal Boyer (2001, pp. 323-328) sur la mort, elles-mêmes inspirées par les travaux de Robert Hertz.

9. Nous laisserons ici de côté la question des périodicités, journalière et saisonnière. Remarquons seulement, d'une part, que la pratique était plutôt estivale, saison oủ les nuits sont brèves, ce qui implique une course contre la montre dans l'accomplissement du rite, d'autre part, que le caractère nocturne est bien de façon privilégiée rattaché à la périodicité journalière. Or nous avions établi la corrélation forte qui prévalait entre elle et la masculinité, laquelle se voit donc ici confirmée (Désveaux 1988, pp. 169-170). 
10. Sur l'intérêt heuristique des quadrants dans le contexte amérindien et la conversion entre groupe de Klein et formule canonique en particulier, nous renvoyons au chapitre II ( ( Groupe de Klein et formule canonique ») de Quadratura Americana (2001).

11. L'ethnologue, qu'il soit culturaliste, structuraliste (lévi-straussien) ou cognitiviste, a désormais coutume de magnifier le savoir naturaliste des Amérindiens. En matière de zoologie, il a peu de peine à montrer la qualité de leurs observations dans le domaine de l'anatomie et de l'éthologie. On notera toutefois que si accouplement, naissance et stades de développement des petits semblent faire l'objet d'un savoir positif extrêmement précis, la question de la longévité des animaux reste totalement ignorée des informateurs. Nous avions posé cette question à nos hôtes de Big Trout Lake, sans obtenir aucun résultat sinon une interrogation en retour sur sa pertinence. Dans son travail monumental consacré aux Montagnais, certainement l'œuvre la plus fouillée dont nous disposons en matière d'ethnozoologie autochtone nord-américaine, Daniel Clément reconnaît qu'il ne dispose d'aucune donnée sur ce point. Ce qui signifie que l'animal possède bien un début - l'accouplement de ses géniteurs -, mais qu'au-delà il est soustrait à la temporalité, que son corps, en tant que substance, n'est pas exposé à ne pas être (au terme d'une période vitale qui lui serait au préalable impartie), car il n'est déjà pas. La mort « naturelle » n'existe pas. Toute mort, celle des humains y compris d'ailleurs, est accidentelle ou, ce qui revient au même, provoquée par une cause «chamanique», nous serait-elle imperceptible dans le contexte d'un perspectivisme généralisé (Clément 1995, p. 344).

12. Voir la section amazonienne de l'argumentaire de l'exposition Qu'est-ce qu'un corps ? présenté en décembre 2002 au musée du quai Branly, par S. Breton, M. Coquet, M. Houseman, J.-M. Schaeffer, A.-C. Taylor et E. Viveiros de Castro. De notre point de vue, on peut se demander s'il ne faut pas lier cette valorisation extrême de la substance au grand raffinement de l'art de la plume : au-delà de sa propension à connoter la transcendance, la plume apparaît également comme le matériau le plus approprié à rehausser avec le plus d'éclat l'apparence de la substance. C'est son habillage idéal.

13. À première vue, nous soutenons ici une position radicalement contraire à celle de Fred Adler (1998). On s'aperçoit toutefois que si son étude à propos des faits africains d'apparence totémique débute dans une veine très classique, presque pré-lévi-straussienne, elle se termine sur des considérations qui ont avant tout trait à l'organisation politique. Il rapporte ces faits à une modalité particulière de la dichotomic, classique sur le continent noir, entre gens de la terre et détenteurs de la royauté.

\section{BIBLIOGRAPHIE}

\section{AdLer Fred}

1998 "Le totémisme en Afrique noire », in Systèmes de pensée en Afrique noire, 15, pp. 13-107, numéro spécial : Totémismes.

\section{BOyer Pascal}

2001

Et l'homme créa les dieux. Comment expliquer la religion? Robert Laffont, Paris.

Breton Stéphane, Michèle Coquet, Michael Houseman, Jean-Marie Schaeffer, AnneChristine TAYlor et Eduardo Viveiros de Castro

2002 Qu'est-ce qu'un corps ? Projet d'exposition dans le cadre du musée du quai Branly, Paris, ms.

\section{Clastres Pierre}

1972 Chronique des Indiens Guayaki, Plon, Paris.

\section{Clément Daniel}

1995 La Zoologie des Montagnais, Peters, Paris. 
DÉsveaux Emmanuel

1988 Sous le signe de l'ours. Mythes et temporalité chez les Ojibwa septentrionaux, Éditions de la Maison des sciences de l'homme, Paris.

2001 Quadratura Americana. Essai d'anthropologie lévi-straussienne, Georg, Genève.

Fogelson Raymond D. et Robert A. Brightman

2002 "Totemism reconsidered", in William L. Merrill et Ives Goddard, éds, Anthropology, history, and American Indians : essays in honor of William Curtis Sturtevant, Smithsonian Institution Press, Washington, pp. 305-313, coll. « Smithsonian contributions to anthropology » 44.

Fowler Catherine $\mathrm{S}$.

1982 «Food-named groups among the northern Paiute in North America's Great Basin ", in Nancy M. Williams et Eugene S. Hunn, éds, Resource managers : North American and Australian hunter-gatherers, Westview Press for the American Association for the advancement of science, Boulder (Colorado), pp. 113-129.

Hallowell Irving A.

1976 Contributions to anthropology : selected papers, University of Chicago Press, Chicago.

Lévi-Strauss Claude

1962 Le Totémisme aujourd'hui, Presses universitaires de France, Paris.

1962 La Pensée sauvage, Plon, Paris.

LowIE Robert H.

1912 "On the principle of convergence in ethnology ", Journal of American Folklore, XXV, pp. 24-42.

Matos Moctezuma Eduardo et Felipe Solis Olguin

2003 Aztecs, Royal Academy of Arts, London [exhibition in Royal Academy of Arts, London, 16 november 2002-11 april 2003].

Merrill William L.

2002 «Species transformations in Northern Mexico : explorations in Rarámuri zoology », in William L. Merrill et Ives Goddard, éds, Anthropology, history, and American Indians : essays in honor of William Curtis Sturtevant, Smithsonian Institution Press, Washington, pp. 333-347, coll. «Smithsonian contributions to anthropology $\gg 44$.

Rosa Frederico

2003 L'Âge d'or du totémisme. Histoire d'un débat anthropologique (1887-1929),

CNRS Éditions/Éditions de la Maison des sciences de l'homme, Paris.

TANNER Adrian

1979 Bringing home animals. Religious ideology and the mode of production of the Mistassini Cree hunters, C. Hurst, London.

Viveiros de Castro Eduardo

2002 A inconstância da alma selvagem : e outros ensalos de antropologia, Cosac \& Naify, Sao Paulo. 